Conference Paper

\title{
Programmable Gesture Manipulator for Hand Injuries Rehabilitation
}

Kozhaeva, T., Zhestkov, S., Bulakh, D. and Houlden, N.

This is a paper presented at the 7th IEEE Int. Conference on Internet Technologies and Applications ITA-17, Wrexham, UK, 12-15 September 2017

Copyright of the author(s). Reproduced here with their permission and the permission of the conference organisers.

\section{Recommended citation:}

Kozhaeva, T., Zhestkov, S., Bulakh, D. and Houlden, N. (2017) 'Programmable Gesture Manipulator for Hand Injuries Rehabilitation'. In: Proc. 7th IEEE Int. Conference on Internet Technologies and Applications ITA-17, Wrexham, UK, 12-15 September 2017, pp. 134-136. doi: 10.1109/ITECHA.2017.8101924 


\section{Programmable Gesture Manipulator for Hand Injuries Rehabilitation}

\author{
Tatyana Kozhaeva, Sergey Zhestkov, Dmitry Bulakh \\ National Research University of Electronic Technology \\ Zelenograd, Moscow, Russia
}

\author{
Nigel Houlden \\ Glyndwr University \\ Plas Coch, Mold Road, Wrexham, LL11 2AW, UK
}

\begin{abstract}
This paper presents a device for hand injury rehabilitation. It allows recognition of different gestures made by a patient and measures such important healing rating as gesture amplitude, movement speed and motion trajectory accuracy. For use with children's rehabilitation it is implemented an ability to control different external devices with different gestures that could be set by using developed software.
\end{abstract}

Keywords-hand injuries rehabilitation, rehabilitation device, gesture recognition, accelerometer gesture recognition, wireless communication

\section{INTRODUCTION}

Hand injuries are frequent types of injuries that may be caused by many reasons such as sports or recreational activities, work-related tasks, accidents and diseases. It is well documented in the literature that an important component in achieving optimal rehabilitation outcome is intensive active movement practice. For hand injury rehabilitation there are several developed devices, most of them are purely mechanical. An example of such devices are shown in Fig. 1a (elbow injuries rehabilitation) and Fig. 1b (finger injuries rehabilitation).

Very specific task is the rehabilitation of children's diseases. There are a lot of rehabilitation devices for children that are using game forms of interaction with children to make rehabilitation more easy, comfortable and fun. And the most popular are devices using PC's for interaction with child patients. An example of this is HandTutor [1]. The HandTutor $^{\mathrm{TM}}$ rehabilitation program focuses on improving fine motor skills, sensory and cognitive impairments together with augmented feedback.

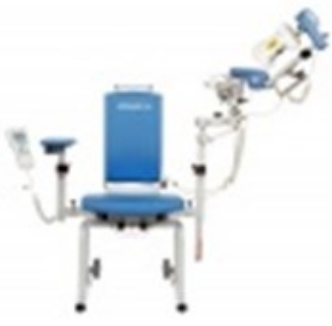

a)

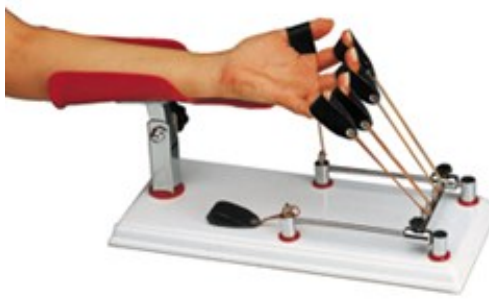

b)
Fig. 1. Mechanical hand injuries rehabilitation devices.
The HandTutor is a glove for fingers and wrist rehabilitation that captures patient gestures with PC software and allows to visualize gestures and measure different gesture characteristics. For children's rehabilitation it uses a specially developed game software that could be controlled by gestures made by patient with gloves. Currently there are no medical devices that are designed to perform gesture rehabilitation for hand injuries to wrist and elbow.

This article presents a developed medical gesture manipulator for wrist and elbow injury rehabilitation, its internal organization and algorithms of its work.

\section{SCHEMES OF THE DEVICES}

Schematic diagram of the developed controller is presented in Fig. 3. The controller device is based on the Arduino platform and consists of Arduino Nano v3.0 microcontroller, digital accelerometer adx1345 and nRF24L01 radio module. The Arduino Nano v3.0 is the main part of the device. It allows to provide interaction between accelerometer and radio module and is programmed using $\mathrm{C}++$ programming language.

The digital accelerometer is used to measure proper acceleration and movement acceleration which occurs while a user performs a gesture. Choosing between analogue

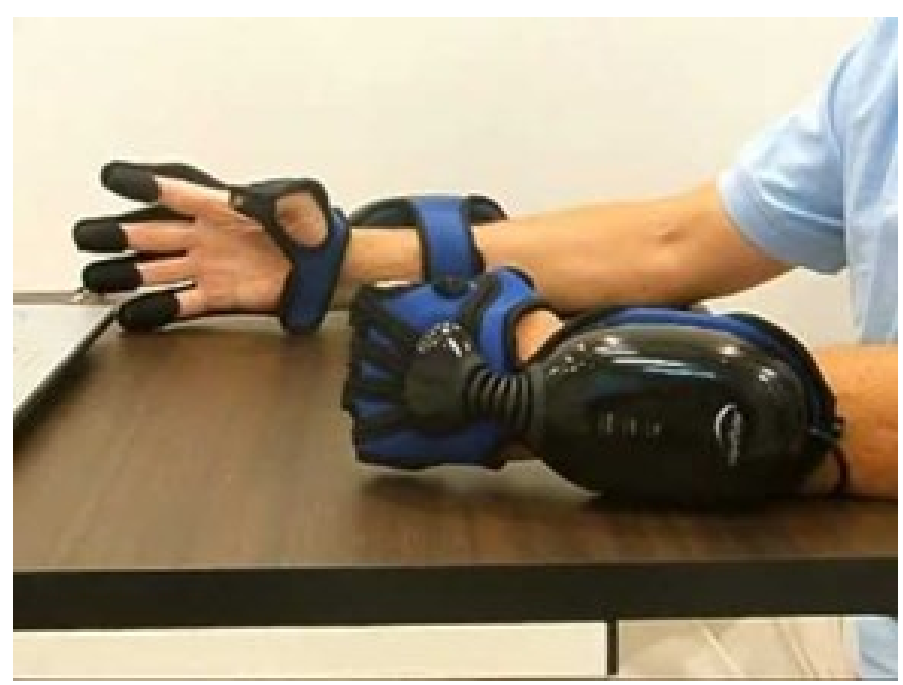

Fig. 2. HandTutor fingers rehabilitation glove. 


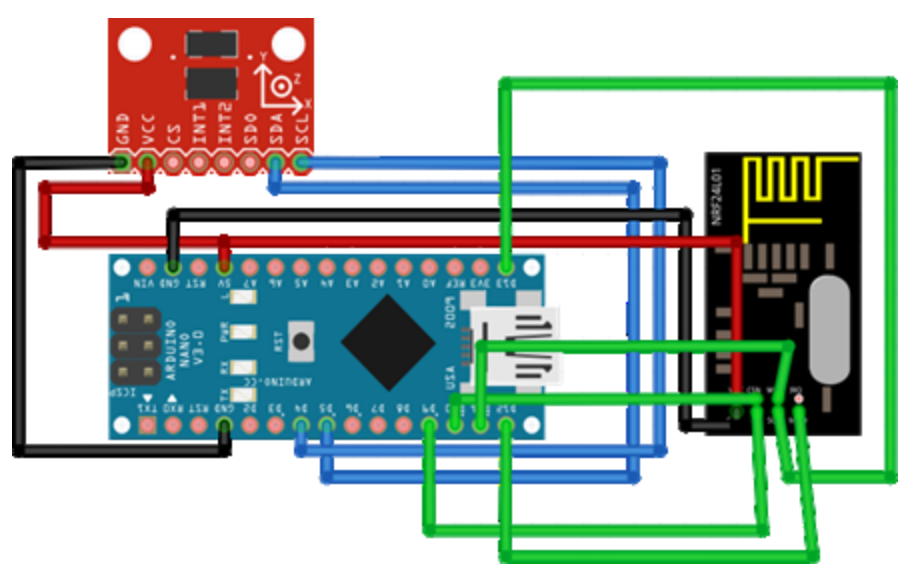

Fig. 3. Arduino based controller device.

TABle I. Wireless Modules EnERgy CONSUMPtion Statistics.

\begin{tabular}{|c|c|}
\hline Module type & Energy consumption (min-max), mA \\
\hline ESP8266 WiFi module & $60-220$ \\
\hline HC-06 Bluetooth module & $30-45$ \\
\hline NRF24L01 radio module & $6-15$ \\
\hline
\end{tabular}

accelerometer adxl335 and digital accelerometer adxl345 digital accelerometer was chosen because of its accuracy and I2C communication interface [2], [3].

To provide wireless communication with controlled devices ESP8266 (WiFi), HC-06 (Bluetooth) and NRF24L01 (radio) modules were tested. Since all of these modules work on the same frequency the first parameter for tests was energy efficiency and the second was distance range. As the result the radio transmitter NRF24L01 was chosen (Table I).

Schematic diagram of any controlled device is shown in Fig. 4. It may use any of microcontroller platform (as an example Arduino UNO R3 was tested), for data communication it has NRF24L01 radio module, for controlling external device $220 \mathrm{~V}$ relay module is used.

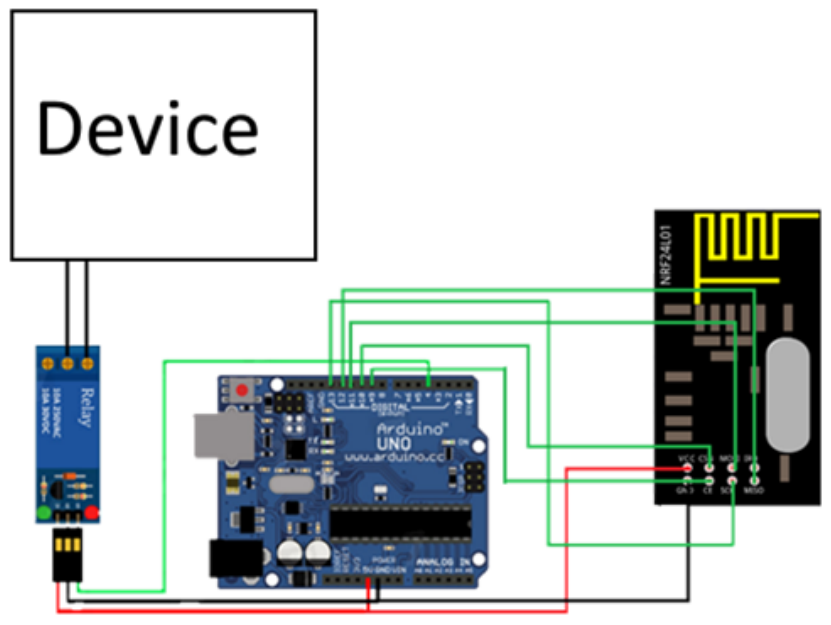

Fig. 4. Schematic diagram of the controller device.

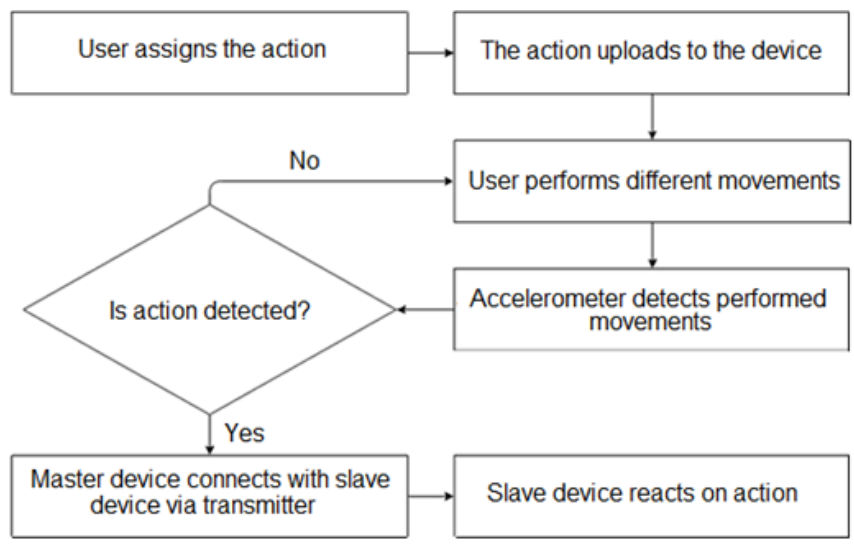

Fig. 5. Basic routine algorithm.

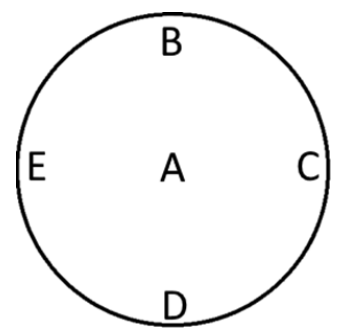

Fig. 6. Checkpoint location map.

\section{GESTURE DETECTION ALGORITHM}

In addition to the schematic solution two algorithms providing control device functionality and its interaction with one or more controlled devices were developed and implemented [4].

The first algorithm is algorithm for gesture detection and recognition, its block diagram is presented in Fig. 5.

The purpose of this algorithm is device movement detection caught by accelerometer. To detect movements, the space in front of controller device is conventionally divided into 5 basic zones: centre, top, bottom, left and right (Fig. 6). Each zone contains checkpoint coordinates attached to it. If accelerometer of controller device passes by any checkpoint it is assumed that the part of gesture was performed and detected.

While passing through the space around the checkpoint it is assumed that action was performed in this direction. Reference coordinates of each checkpoint were defined experimentally. If the accelerometer detects coordinates in the area of any checkpoint with error value 50 , then it's believed that user has performed a movement in a checkpoint's direction.

Information about each gesture is collected in internal memory of microcontroller including such parameters as movement speed, required trajectory deviation and others. All this information is transmitted to the receiver connected to a $\mathrm{PC}$ or in case of using remote controlled devices information is passed to the controlled device.

Besides the specified 5 zones, an additional 4 zones could be implemented to provide more complex gestures. However, 


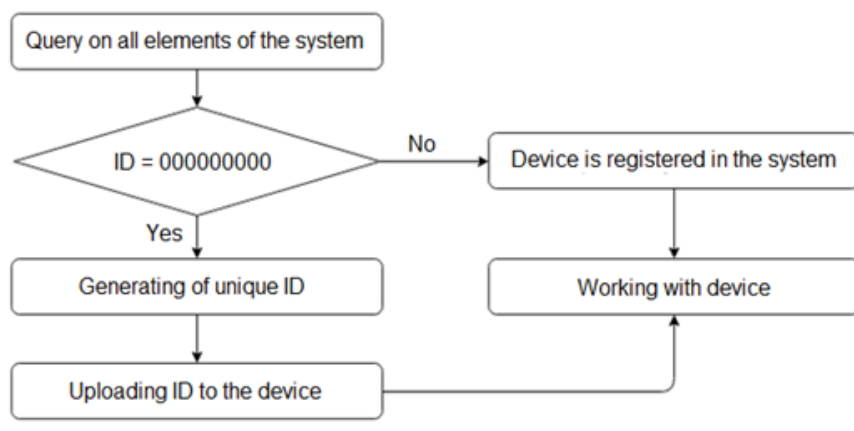

Fig. 7. Device identification and system functioning algorithm.

usage of additional checkpoints makes the gesture detection algorithm more complicated because detection of these checkpoints forces use of specific trigonometric equations.

The given algorithm exposes another task. The problem is that the gesture detection algorithm works correctly only when the control device has a certain orientation in space. If user a will turn the device on any angle around its own axis, then the accelerometer detects wrong coordinates in same checkpoints. This is the reason why special correction coefficients were implemented, which allow the device to use this algorithm without any dependence from its orientation in space.

The second algorithm is an algorithm for providing connection between controller controlled devices [5] which could be connected to PC or even could be absolutely autonomous. The problem was that using wireless communication controlling many devices should be implemented.

The general idea of solving this problem is unique identification of all devices in the system. The most obvious solution is generating and uploading of unique ID for every controlled device. In this case, a control device can recognize which device it should connect to after performing a gesture with ease. Simplified diagram of this algorithm is shown in Fig. 7.

All slave devices in the system have ID $=000000000$ by default. During the first start-up of the hardware and software system control device performs query on all devices in the system and reads their ID. Every found device with $\mathrm{ID}=0000000000$ will be given a new unique nine-digit

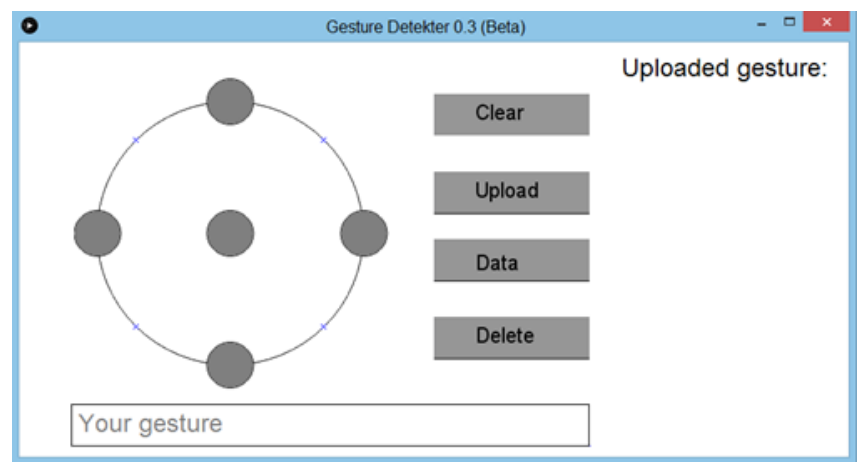

Fig. 8. Gesture definition application user interface. identification number which was automatically generated on the PC in the real time. This new ID will be uploaded on the device via radio channel through control device. The length of ID is caused by restrictions in nRF library which is used to provide interaction between microcontroller and radio module. These IDs will be unique for every similar system. The choice of algorithm which will create a random nine-digit sequence of numbers will be made on the later stages of work.

This developed algorithm solves the problem of adding a lot of new devices in any similar system at any moment of time. Considering that every device has a unique identification number communication between devices with this generated IDs will look like this.

Radio transmitters of all controlled devices in the system are set in data receiving mode on the one channel which was defined earlier. The first data frame in the packet will be unique ID, so this packed will be received only by device with the same identification number and will be ignored by the others.

\section{Gesture Definition Software}

To allow a user to work with the control device a specialized desktop application has been developed. The interface of this software is shown in Fig. 8. The application was implemented using Processing programming language.

Main functionality of this application is associated with the idea of gesture detection algorithm. The software allows to define any sequence of movements to control a specific device using mouse clicks on checkpoints located on the circle. In the text label at the bottom of the window user can see his created gesture in form of sequence of numbers which are mapped with specific directions.

At the present time work on desktop version of this application is in progress. It will be rewritten using $\mathrm{C}++$ programming language with Qt cross-platform GUI library.

\section{CONCLUSION}

In this work of a gesture controlled device, the model of controlled device, a set of algorithms and software including firmware and desktop application were developed. Performed tests and trials have shown the efficiency and suitability of developed device complex in some medical cases.

\section{REFERENCES}

[1] E. Carmeli, S. Peleg, G. Bartur, E. Elbo, and J.-J. Vatine, "Handtutor enhanced hand rehabilitation after stroke - a pilot study," Physiotherapy Research International, vol. 16, no. 4, pp.191-200, Dec 2011.

[2] http://www.analog.com/en/products/mems/accelerometers.html

[3] N. Zhao "Full-featured pedometer design realized with 3-axis digital accelerometer," Analogue Dialogue, vol. 44, no. 6, pp. 1-5, June 2010.

[4] S.G. Zhestkov, and T.D. Kozhaeva, "Development of device for gesture manipulator baseod on ATmega328 microcontroller, " in Proc. MIET Conf. on Microelectronics and Informatics, Moscow, 2016, p. 114. (In Russian)

[5] S.G. Zheskov, and T.D. Kozhaeva, "Development of hardware and software complex for controlling electrical devices," presented at Scientific Fair RITM, MIET, Oct. 2016. (In Russian) 